\title{
Genetic Manipulation of Intraspinal Plasticity after Spinal Cord Injury Alters the Severity of Autonomic Dysreflexia
}

\author{
Adrian A. Cameron, ${ }^{1}$ George M. Smith, ${ }^{1,2}$ David C. Randall, ${ }^{1,2}$ David R. Brown, ${ }^{2}$ and Alexander G. Rabchevsky ${ }^{1,2}$ \\ ${ }^{1}$ Spinal Cord and Brain Injury Research Center and ${ }^{2}$ Department of Physiology, University of Kentucky, Lexington, Kentucky 40536
}

Severe spinal cord injuries above mid-thoracic levels can lead to a potentially life-threatening hypertensive condition termed autonomic dysreflexia, which is often triggered by painful distension of pelvic viscera (bladder or bowel) and consequent sensory fiber activation, including nociceptive $\mathrm{C}$-fibers. Interruption of tonically active medullo-spinal pathways after injury causes disinhibition of thoracolumbar sympathetic preganglionic neurons, and intraspinal sprouting of nerve growth factor (NGF)-responsive primary afferent fibers is thought to contribute to their hyperactivity. We investigated spinal levels that are critical for eliciting autonomic dysreflexia using a model of noxious colorectal distension (CRD) after complete spinal transection at the fourth thoracic segment in rats. Post-traumatic sprouting of calcitonin gene-related peptide (CGRP)-immunoreactive primary afferent fibers was selectively altered at specific spinal levels caudal to the injury with bilateral microinjections of adenovirus encoding the growth-promoting NGF or growth-inhibitory semaphorin 3A (Sema3a) compared with control green fluorescent protein (GFP). Two weeks later, cardio-physiological responses to CRD were assessed among treatment groups before histological analysis of afferent fiber density at the injection sites. Dysreflexic hypertension was significantly higher with NGF overexpression in lumbosacral segments compared with GFP, whereas similar overexpression of Sema3a significantly reduced noxious CRD-evoked hypertension. Quantitative analysis of CGRP immunostaining in the spinal dorsal horns showed a significant correlation between the extent of fiber sprouting into the spinal segments injected and the severity of autonomic dysreflexia. These results demonstrate that site-directed genetic manipulation of axon guidance molecules after complete spinal cord injury can alter endogenous circuitry to modulate plasticity-induced autonomic pathophysiology.

Key words: nerve growth factor; semaphorin 3A; sprouting; sympathetic; neurotrophin; autonomic

\section{Introduction}

Autonomic dysreflexia has an incidence of up to $70 \%$ after clinically complete spinal cord injury above the sixth thoracic $(\mathrm{T})$ vertebral level (T6). It is manifested by often debilitating hypertension accompanied by bradycardia, sweating, skin flushing, and pounding headaches that are commonly triggered by unpleasant distension of pelvic viscera (bowel and bladder) (Snow et al., 1978; Lindan et al., 1980; Harati, 1997; Karlsson, 1999). The etiology of this syndrome likely stems from injury-induced loss of descending tonic, and baroreceptor-related, control of sympathetic preganglionic neurons in the intermediolateral cell column (IML) of the thoracolumbar spinal cord (Finestone and Teasell, 1993; Zagon and Smith, 1993). Concomitant postinjury sensory

\footnotetext{
Received March 21, 2005; revised Nov. 22, 2005; accepted Jan. 25, 2006.

This work was supported by grants from the International Spinal Research Trust, the Kentucky Spinal Cord and Head Injury Research Trust, and the National Institutes of Health-National Institute of Neurological Disorders and Stroke (R01 NS-049901-01) to A.G.R. We thank Drs. Lynne Weaver, Andrei Krassioukov, and Lawrence Schramm for helpful discussions concerning this study. We also express gratitude to Janna Hackett, Peiti Cook, Julie Ter Beest, Kelly Miller, Kainath Durre, Johnna Shipp, Igor Voskresensky, Leslie Schwindel, Travis Lyttle, and Charles Mashburn for expert technical assistance.

Correspondence should be addressed to Dr. Alexander G. Rabchevsky, University of Kentucky, Spinal Cord and Brain Injury Research Center, B371, Biomedical and Biological Sciences Research Building, 741 South Limestone Street, Lexington, KY 40536-0509. E-mail: AGRab@uky.edu.

A. A. Cameron's present address: The Bionic Ear Institute, University of Melbourne, 384-388 Albert Street, East Melbourne, Victoria 3002, Australia.

DOI:10.1523/JNEUROSCI.4390-05.2006

Copyright $\odot 2006$ Society for Neuroscience $\quad 0270-6474 / 06 / 262923-10 \$ 15.00 / 0$
}

fiber sprouting and the afferent barrage into spinal cord dorsal horns during noxious distension of pelvic viscera are thought to elicit unopposed reflex sympathetic discharge below the injury level, resulting in vasoconstriction of the muscular, splanchnic, and cutaneous vascular beds (for review, see Karlsson, 1999). The resultant paroxysmal hypertension produces a baroreceptormediated reflex bradycardia accompanied by withdrawal of sympathetic activity above the injury level with consequential vasodilatation producing adverse symptoms such as headaches.

Using a rodent spinal cord injury model of inducing autonomic dysreflexia with noxious colorectal distension (CRD) to mimic clinical manifestations of fecal impaction (Krassioukov and Weaver, 1995), we have verified that a contributing factor is injury-induced elevation in spinal levels of nerve growth factor (NGF) and subsequent intraspinal sprouting of calcitonin generelated peptide-immunoreactive $\left(\mathrm{CGRP}^{+}\right)$, NGF-responsive sensory afferents (Krenz and Weaver, 1998; Krenz et al., 1999; Weaver et al., 2001; Marsh et al., 2002; Brown et al., 2004). Importantly, CRD evokes electrical responses of thoracic dorsal horn neurons, which are correlated with enhanced activity in postganglionic sympathetic neurons (Chau et al., 2000). We reasoned, therefore, that injury-induced sprouting of sensory afferents from the distal colon is relayed from their entry into lumbosacral segments (Pascual et al., 1993; Wang et al., 1998; Vizzard, 2000) to thoracic levels by enhanced activity of propriospinal projections that stimulate sympathetic preganglionic neurons and/or sympathetically related dorsal horn interneurons. 
The principle goal of this study was to manipulate critical sites instrumental in the production of dysreflexic autonomic responses to noxious CRD. We specifically altered injury-induced $\mathrm{CGRP}^{+}$afferent fiber sprouting in dorsal horns of selected spinal levels caudal to a complete T4 spinal cord transection with microinjections of replication-defective, temperature-sensitive adenoviruses (Adts) encoding the growth-promoting NGF (NGF Adts) (Romero et al., 2000, 2001) or the sympathetic and C-fiber growth-inhibitory semaphorin 3A (Sema3a Adts) (Tang et al., 2004). Two weeks later, injured animals were tested for cardiophysiological responses to noxious CRD (Maiorov et al., 1998) with subsequent immunohistochemical analyses of CGRP ${ }^{+}$fiber density in the dorsal horns (Krenz et al., 1999; Weaver et al., 2001). In summary, we found that overexpression of chemorepulsive Sema3a in lumbosacral segments receiving afferent input from the distal colon significantly reduced $\mathrm{CGRP}^{+}$sprouting and mitigated the severity of hypertensive autonomic dysreflexia. The opposite conditions occurred with NGF overexpression, demonstrating that axon guidance molecules can alter endogenous circuitry to modulate the severity of plasticity-induced autonomic pathophysiology after spinal cord injury.

\section{Materials and Methods}

Surgeries and injections of adenovirus and FluoroGold. All animal housing conditions, surgical procedures, and postoperative care were conducted according to our University of Kentucky Institutional Animal Care and Use Committee-approved protocol and National Institutes of Health animal care guidelines. Adult female Wistar rats $(n=59 ; 250 \mathrm{~g})$ were anesthetized with a mixture of ketamine $(80 \mathrm{mg} / \mathrm{kg}$, i.p.; Fort Dodge Animal Health, Fort Dodge, IA) and xylazine (7 mg/kg, i.p.; Butler, Columbus, $\mathrm{OH}$ ) before a complete laminectomy was performed on the T3 vertebra to expose the T4 spinal segment. Another partial laminectomy was then performed at the T5 vertebrae to expose the T5/6 segment ( $n=$ $5)$, the T11/T12 vertebrae to expose the T13/L1 segment $(n=14)$, or at the L1/L2 vertebrae to expose the L6/S1 segment $(n=31)$ for adenoviral injections (see Table 1). These levels were chosen because T5/6 is the middle of the IML-containing sympathetic preganglionic neurons projecting to visceral vascular beds, whereas T13/L1 and L6/S1 are the sites of termination of primary afferents innervating the distal colon (Pascual et al., 1993; Wang et al., 1998; Vizzard, 2000). After the laminectomies, a complete transection of the T4 segment was performed with a scalpel blade, confirmed both visually at the time of transection and when the spinal cord was dissected after perfusion. To inject Adts encoding NGF, green fluorescent protein (GFP), or Sema3a, glass micropipettes with tip diameters of $30-50 \mu \mathrm{m}$ were lowered into the spinal dorsal horn $500 \mu \mathrm{m}$ from the surface and $300 \mu \mathrm{m}$ from the midline. For each adenoviral vector, $500 \mathrm{nl}$ of $5 \times 10^{6} \mathrm{pfu} / \mu \mathrm{l}$ was pressure injected bilaterally in $50 \mathrm{nl}$ pulses over a period of 2 min using a nano-injector (World Precision Instruments, Sarasota, FL). The pipette was left in place an additional 4 min to minimize leakage of the virus during withdrawal of the needle. In designated animals, sympathetic preganglionic neurons were prelabeled with FluoroGold (hydroxystilbamidine, methanesulfonate; Biotium, Hayward, CA) by injecting $0.2 \mathrm{ml}$ of a $1.5 \%$ solution intraperitoneally 1 week after injury (Anderson and Edwards, 1994). These animals were then perfused with fixative after physiological testing 1 week later (see below). Three additional rats were used as naive, uninjured controls for cardio-physiological responses to noxious CRD. Sham-injured rats $(n=$ 9) were similarly injected at L6/S1 with NGF Adts, GFP Adts, or Sema3a Adts for Western blot verification of transgene protein expression $(n=3$ per group), as described previously (Tang et al., 2004).

After intraspinal injections were complete, the erector spinae muscle was sutured with Vicryl (Ethicon, Sommerfield, NJ), the field was disinfected with povidone-iodine solution (Nova Plus, Irving, TX), and the skin was closed with Michel wound clips (Roboz, Gaithersburg, MD). Rats were administered $20 \mathrm{ml}$ of lactated Ringer's solution subcutaneously (Baxter Healthcare, Deerfield, IL) and $33 \mathrm{mg} / \mathrm{kg}$ cephazolin (Apothecon, Princeton, NJ) subcutaneously immediately after surgery and twice daily for $10 \mathrm{~d}$ to maintain hydration and control infection. Buprenorphine $(0.035 \mathrm{mg} / \mathrm{kg}$, s.c.; Reckitt Benckiser, Berks, UK) was administered the next day to control postoperative pain. Bladders were expressed manually twice daily until the animals were killed.

Vector construct. The coding regions for NGF or GFP were inserted into a modified pXCJL vector (gift from Dr. Frank Graham, McMaster University, Hamilton, Ontario, Canada) containing the Rous sarcoma virus long terminal repeat and the bovine growth hormone polyadenylation. A flag peptide epitope was inserted at the $\mathrm{C}$ terminus of the NGF cassette to facilitate discrimination from endogenous molecules. Recombinant temperature-sensitive Adts were generated, plaque-purified, and isolated on a cesium chloride gradient as described previously (Romero and Smith, 1998). Replication-defective Sema3a-expressing Adts was constructed as described previously (He et al., 1998). The coding region of Sema3a with a Myc-epitope tag (a gift from Dr. Corey S. Goodman, University of California at Berkeley, Berkeley, CA) was inserted into the pAdtrack-GFP vector. All recombinant adenovirus was generated using the AdEasy system (He et al., 1998), in which the vector was modified to include a temperature-sensitive mutation $(t s 125)$ within the DNAbinding protein of adenovirus (Romero and Smith, 1998). After transfection of human embryonic kidney 293 cell lines, virus production was monitored by GFP expression and plaque formation. Adts were amplified and purified on a cesium-chloride gradient as described previously (Romero and Smith, 1998). The viral particle to plaque-forming unit (pfu) ratios for these viruses was $<55: 1$, as determined using Adeno-X rapid titer kit (Clontech, Mountain View, CA).

Assessing autonomic dysreflexia with colorectal distension. Fourteen days after spinal transection, femoral cannulas were implanted in rats to test dysreflexia the following day, as described previously (Maiorov et al., 1997). Briefly, animals were reanesthetized (ketamine, $80 \mathrm{mg} / \mathrm{kg}$; xylazine, $7 \mathrm{mg} / \mathrm{kg}$ ), and the femoral artery was exposed and catheterized with beveled polyethylene (PE-60) tubing (Becton-Dickinson, Sparks, MD) filled with heparinized saline $(1000 \mathrm{U} / \mathrm{ml})$. The peripheral end was tunneled under the skin to the nape leaving $\sim 1$ inch protruding to plug with a removable, blunt T-shaped 23 gauge steel needle. Blood pressure was immediately verified by attaching a Transpac pressure transducer (Abbott Labs, Chicago, IL) and a Grass preamplifier that interfaced with a computer equipped with an arterial pressure acquisition program (ViiSoftware, Lexington, KY). After the initial recording (and $12 \mathrm{~h}$ later), the catheter was flushed with heparinized saline and the steel plug replaced. On the following day after full recovery from anesthesia, the heart rate (HR), systolic pressure, diastolic pressure, and mean arterial pressure (MAP) were monitored under experimental conditions and displayed using a PowerLab (ADInstruments, Castle Hill, Australia) system, which digitizes information from the amplifiers and stores it on a computer hard drive.

To initiate spinal viscero-sympathetic reflexes, a latex balloon-tipped catheter (Swan-Ganz; Edwards LifeSciences, Irvine, CA) was inserted 2 $\mathrm{cm}$ inside the rectum, secured to the tail with tape, and left in place until the MAP stabilized, indicating the animal's habituation (Maiorov et al., 1997). The MAP was then measured before (baseline), during, and after a $1 \mathrm{~min}$ period of balloon catheter inflation with $2 \mathrm{ml}$ of air to distend the distal colon designed to mimic noxious fecal impaction. Three trials were performed in quiet surroundings the day after catheterization on all animals, $\sim 30$ min apart. An animal was regarded as dysreflexic if CRD produced a rise in MAP and a decrease in HR for as long as the period of CRD. In addition the uninjured, untreated control rats were catheterized for measurement of noxious CRD-induced MAP responses in intact animals. For each animal, the difference between baseline MAP and CRDinduced MAP change was first calculated for each trial and then averaged over the three trials. These values were further averaged for each of the three treatment groups (GFP Adts, NGF Adts, Sema3a Adts) across the three spinal levels chosen to inject with Adts.

Western blot analysis. Two weeks after injections, the L6/S1 segment of sham-injured rats injected with NGF Adts, GFP Adts, or Sema3a Adts were processed for Western blot analysis ( $n=3$ per group) as described previously (Tang et al., 2004). Briefly, a $4 \mathrm{~mm}$ segment of the spinal cord containing the injected region was dissected and immediately frozen. The tissue was then homogenized manually with a dounce in $200 \mu \mathrm{l}$ of $1 \%$ 
Table 1. MAP and HR before (baseline) and during CRD in naive uninjured rats and those with $\mathrm{T} 4$ spinal cord transactions with subsequent intraspinal injections of adenovirus encoding GFP (control), NGF, or Sema3a at specified spinal levels

\begin{tabular}{lllll}
\hline Treatment/spinal level & $\begin{array}{l}\text { MAP baseline } \\
(\mathrm{mmHg} \pm \text { SD) }\end{array}$ & $\begin{array}{l}\text { HR baseline } \\
(\mathrm{bpm} \pm \text { SD })\end{array}$ & $\begin{array}{l}\text { MAP CRD } \\
(\mathrm{mmHg} \pm \text { SD) }\end{array}$ & $\begin{array}{l}\text { HR CRD } \\
(\mathrm{bpm} \pm \text { SD) }\end{array}$ \\
\hline Naive $(n=3)$ & $111 \pm 10$ & $450 \pm 80$ & $35.4 \pm 4$ & $+76.2 \pm 22$ \\
NGF Adts/T5/6 $(n=5)$ & $106 \pm 7$ & $540 \pm 50$ & $22.0 \pm 4$ & $-25.8 \pm 12$ \\
GFP Adts/ T13/L1(n=5) & $106 \pm 5$ & $477 \pm 92$ & $22.5 \pm 7$ & $-33.6 \pm 24$ \\
NGF Adts/ T13/L1 $(n=9)$ & $104 \pm 8$ & $475 \pm 88$ & $32.7 \pm 5$ & $-43.8 \pm 24$ \\
GFP Adts/ L6/S1 $(n=9)$ & $105 \pm 8$ & $511 \pm 86$ & $21.1 \pm 5$ & $-29.6 \pm 20$ \\
NGF Adts/ L6/S1 $(n=8)$ & $102 \pm 8$ & $472 \pm 94$ & $30.5 \pm 5$ & $-40.8 \pm 27$ \\
Sema3a Adts/ L6/S1 ( $n=14)$ & $101 \pm 10$ & $499 \pm 89$ & $14.1 \pm 5$ & $-30.6 \pm 18$ \\
\hline
\end{tabular}

SDS in Tris-EDTA buffer with proteinase inhibitors ( $10 \mu \mathrm{g} / \mathrm{ml}$ aprotinin, $1 \mu \mathrm{g} / \mathrm{ml}$ leupeptin, and $1 \mathrm{~mm}$ PMSF) and sonicated using a Branson sonifier 450 (VWR Scientific, West Chester, PA). After centrifuging at $14,000 \mathrm{rpm}$, supernatant was assayed for protein concentration using a BCA kit (Pierce, Rockford, IL), diluted with $3 \times$ Laemmli's buffer, and 50 $\mu \mathrm{g}$ of protein loaded for each sample. After running the sample in $14 \%$ (for NGF), 10\% (for GFP), or 7.5\% (for Sema3a) SDS-polyacrylamide gel, proteins were transferred to nitrocellulose membranes. Membranes were blocked using Odyssey (LI-COR, Biosciences, Lincoln, NE) blocking buffer. NGF was identified by rabbit anti-NGF antibody (1:500; Accurate Chemicals, Westbury, NY), and adenovirus-mediated Sema3a expression was detected by mouse anti-Myc (1:2000; Upstate, Charlottesville, VA). After a $2 \mathrm{~h}$ incubation in primary antibody, the membranes were washed five times for $10 \mathrm{~min}$ in Tris-buffered saline with $0.1 \%$ Tween 20 and incubated in goat anti-rabbit (for NGF and GFP) or mouse (for Sema3a) IgG (1:7500; Invitrogen, Carlsbad, CA) conjugated with AlexaFluor 680 for $1 \mathrm{~h}$. Membranes were washed as above, and images were captured using an Odyssey infrared imaging system (LI-COR Biosciences) at $700 \mathrm{~nm}$.

Fixation and immunohistochemistry. After completion of the physiological testing, animals were overdosed with sodium pentobarbital (150 $\mathrm{mg} / \mathrm{kg}$; Abbott Labs) and perfused through the aorta with $0.1 \mathrm{M}$ PBS, $\mathrm{pH}$ 7.4 , followed by $4 \%$ paraformaldehyde in PBS. The spinal cord from the conus medullaris to the transection site was removed, postfixed for $3 \mathrm{~h}$, rinsed in $0.2 \mathrm{M}$ phosphate buffer, cryoprotected for $48 \mathrm{~h}$ in $20 \%$ sucrose in PBS, cryosectioned coronally at $20 \mu \mathrm{m}$, and mounted on glass slides (Superfrost Plus; Fisher Scientific, Pittsburgh, PA). For stereological sampling (Rabchevsky et al., 2001, 2002), the cords were divided into two, $3 \mathrm{~cm}$ portions. The caudal segment encompassed spinal levels S4 to $\mathrm{T} 13$ and the rostral segment was from T12 to T5. For both segments, 20 $\mu \mathrm{m}$ cryosections taken every $100 \mu \mathrm{m}$ were mounted onto one of two series of 10 slides, consecutively (1-10 thoracolumbar; 1a-10a lumbosacral). Up to six cords were evenly aligned in a row and embedded in each tissue block, and up to 15 consecutive rows of cryosections from each block were placed onto each of 10 slides in both series. Such processing results in parallel columns of adjacent mounted cryosections from six different cords each separated by $1 \mathrm{~mm}$ mounted on each slide of 10 in both series that represent equal rostrocaudal dissected segments. In this manner, if $0.5 \mathrm{~mm}$ separation is desired, then two slides separated by 5 in the series of 10 are processed for histological analysis (i.e., slides 1 and 6, $1 \mathrm{a}$ and $6 \mathrm{a})$.

For immunohistochemistry, sections were preincubated in PBS containing $5 \%$ normal goat serum and $0.2 \%$ Triton- $\mathrm{X}$ for $30 \mathrm{~min}$ at room temperature. They were then incubated overnight at $4^{\circ} \mathrm{C}$ with rabbit anti-CGRP (Chemicon, Temecula, CA) diluted 1:2000 or substance P (SP) diluted 1:500 (Sigma, Natick, MA) in PBS containing 5\% normal goat serum and $0.2 \%$ Triton-X. After rinsing in PBS, the sections were incubated for $3 \mathrm{~h}$ at room temperature in PBS containing biotinylated goat anti-rabbit antibody (diluted 1:400 in PBS containing 5\% normal goat serum and $0.2 \%$ Triton-X; Vector Laboratories, Burlingame, CA). After rinsing in PBS, the sections were incubated for another $3 \mathrm{~h}$ at room temperature in PBS containing streptavidin-Texas Red (diluted 1:200; Vector Laboratories). The Sema3a vector also contained a promoter for GFP, which enabled verification of both GFP and Sema3a Adts injection sites for colocalization with CGRP and SP staining. NGF Adts injection sites demonstrated copious $\mathrm{CGRP}^{+}$fiber sprouting as we reported previously (Romero et al., 2000, 2001). To verify this, mouse anti-FLAG (M2 antibody; diluted 1:100; Stratagene, La Jolla, CA) was coincubated with rabbit antiCGRP and revealed with FITCconjugated donkey anti-mouse (1: 100; Jackson ImmunoResearch, West Grove, PA). Sections showing prominent immunoreactivity for exogenous FLAG M2 were designated for quantification of CGRP or SP-Texas Red immunostaining in the dorsal horns (see below). After final rinses in PBS, the sections were coverslipped with Vectashield medium (Vector Laboratories) and sealed with Cutex (Jackson, WY) nail hardener.

Quantitative image analysis. Sections were examined on an Olympus (Melville, NY) BX51 microscope. Photos were taken with an Olympus Magnafire digital camera, and images were quantified using Bioquant image analysis (Nova Prime, version 6.70.10; Bioquant Image Analysis, Nashville, TN). All sections were photographed with the same objective magnification $(4 \times ; 10 \times$ eye piece) and with the same exposure settings. In all sections analyzed, the region of interest to quantify the area of $\mathrm{CGRP}^{+}$or $\mathrm{SP}^{+}$immunoreactivity incorporated both dorsal horns including the gray matter just ventral to the central canal (see Fig. 6). For each animal, regardless of treatment, three serial sections centering on the injection site and separated by $1 \mathrm{~mm}$ were selected for $\mathrm{CGRP}^{+}$or $\mathrm{SP}^{+}$areal quantification to account for the rostrocaudal spread of Adts, which can be as much as $5 \mathrm{~mm}$ with $500 \mathrm{nl}$ injections of GFP Adts (Cameron et al., 2004; Tang et al., 2004) and $\beta$-galactosidase Adts (Romero and Smith, 1998). For animals that received GFP Adts or Sema3a Adts, the injection sites were identified by locating the section in which GFP expression appeared the greatest, and NGF Adts injection sites were identified by widespread immunoreactivity for FLAG. To measure the area of CGRP ${ }^{+}$or SP $^{+}$immunostaining occupying the regions of interest, $\mathrm{CGRP}^{+}$or $\mathrm{SP}^{+}$fibers were "thresholded" on each digital photograph, using the Bioquant program. For each section, the percentage of coverage of $\mathrm{CGRP}^{+}$or $\mathrm{SP}^{+}$fibers was calculated as the ratio of bilateral immunoreactive area/region of interest area in dorsal gray matter $\times 100$. This calculation was made to compensate for the different size of the regions of interest at different spinal levels (e.g., lumbar vs sacral). The mean percentage of coverage over the three sections in each animal was calculated, followed by the mean percentage of coverage for all animals in a treatment group.

Statistical analysis. For measurements of CRD-evoked changes in MAP and percentage of $\mathrm{CGRP}^{+}$areal coverage in the dorsal horns, significance differences between treatment groups were determined by ANOVA across treatments and spinal levels followed by unpaired $t$ tests with Bonferroni's correction factor when appropriate, using Statview 5.0 (SAS Institute, Cary, NC). All slides were assessed blindly with respect to treatment. Linear regression analysis correlated percentage of CGRP ${ }^{+}$ fiber areal coverage in the dorsal horns with CRD-induced MAP changes. Significance throughout all experiments was set at $p<0.05$. Graphs in the figures depict group mean values, and error bars represent SD.

\section{Results}

\section{Autonomic dysreflexia: noxious CRD-induced changes in MAP and HR}

Physiological measurements of controls

The baseline MAP and HR ( $\pm \mathrm{SD})$ before noxious CRD in conscious uninjured rats and those with $\mathrm{T} 4$ spinal transection and Adts injections 2 weeks previously showed no significant differences across treatment groups (Table 1). In spinal-intact, awake animals $(n=3)$, we confirmed that noxious CRD with balloon catheter inflation produced characteristic (Maiorov et al., 1998) MAP increases of $35.4 \pm 4 \mathrm{mmHg}$ accompanied by HR increases 
of $76.2 \pm 22$ beats per minute (bpm), followed by a rapid return to baseline levels after balloon deflation indicative of an intact normal baroreflex (Fig. 1A). Conversely, CRD performed 2 weeks after T4 spinal transection in animals injected with control GFP Adts at either T13/L1 $(n=5)$ or L6/S1 $(n=9)$ elicited MAP increases of $\sim 22 \mathrm{mmHg}$ accompanied by reduced HR of $\sim 32 \mathrm{bpm}$, which is characteristic of autonomic dysreflexia (Figs. 1B, 2).

Physiological responses to intraspinal NGF versus Sema3a overexpression

NGF and Sema3a Adts were injected into specified regions of the injured spinal cord to examine whether localized overexpression would differentially alter $\mathrm{CGRP}^{+}$fiber sprouting and the severity of autonomic dysreflexia. An ANOVA across the treatment groups (GFP Adts, NGF Adts, Sema3a Adts) and spinal levels (T5/6, T13/L1, L6/S1) revealed a signifi-

cant difference in CRD-induced MAP increases $15 \mathrm{~d}$ after T4 transection $\left(F_{(5,44)}=17.531 ; p<0.001\right)$. To determine whether localized NGF expression had a direct influence on sympathetic preganglionic neuron activity, injections of NGF Adts were made at thoracic T5/6 $(n=5)$ to induce sprouting into the IML. Such injections rendered CRD-induced hypertension (22.0 \pm 4 $\mathrm{mmHg})$ and bradycardia $(25.8 \pm 12 \mathrm{bpm})$ that were equal to those observed in injured GFP Adts-injected controls (Fig. 2). Although local NGF overexpression did not directly influence the sympathetic discharge in response to CRD, this does not dismiss the possibility that noxious stimuli applied to the trunk would augment autonomic dysreflexia. However, these somewhat predictable results verified that increased spasms, vasoconstriction, and piloerection below the injury elicited by CRD did not secondarily activate hypersensitive T5/6 dermatome afferents that sprouted into the high thoracic IML. Therefore, based on the evidence that T13/L1 and L6/S1 are the sites of termination of primary afferents innervating the distal colon (Pascual et al., 1993; Wang et al., 1998; Vizzard, 2000), we manipulated injuryinduced sprouting at these more caudal levels. We found that NGF overexpression at T13/L1 $(n=9)$ or L6/S1 $(n=8)$ significantly increased CRD-induced hypertension to $\sim 32 \mathrm{mmHg}$ above baseline, accompanied by bradycardia of $\sim 42 \mathrm{bpm}$ (Figs. $1 C, 2)$.

Because NGF Adts promote robust sprouting of primary afferent $\mathrm{CGRP}^{+}$fibers (Romero et al., 2001), these observations suggested that $\mathrm{C}$-fiber sprouting into the lumbosacral spinal cord may be responsible for significant increases in hypertension during noxious CRD in animals receiving injections of NGF Adts at T13/L1 or L6/S1. Accordingly, growth-inhibitory Sema3a was overexpressed at L6/S1 $(n=14)$ to inhibit local spontaneous fiber sprouting (Tang et al., 2004). When compared with injured rats injected with GFP Adts at L6/S1, Sema3a Adts overexpression significantly reduced noxious CRD-evoked hypertension to $14.1 \pm 5 \mathrm{mmHg}$ above baseline with bradycardia of $30.6 \pm 18$ bpm (Figs. 1D, 2). Thus, autonomic dysreflexia was not eliminated by local overexpression of Sema3a, but the severity of hypertension was significantly less than in control injured rats. Although MAP changes were significantly different after NGF and Sema3a overexpression compared with injured controls, the nominal disparity in MAP measurements within experimental groups was reflected in a high degree of $\mathrm{HR}$ variability (Fig. $2 \mathrm{~B}$ ).

\section{Dorsal horn immunohistochemistry for CGRP ${ }^{+}$and $\mathrm{SP}^{+}$fibers \\ Verification of injection sites}

Bilateral injection sites for the different Adts constructs were identified for immunohistochemical analysis by examining the expression of either GFP for both the controls and Sema3a Adts or immunoreactivity for the FLAG epitope of NGF Adts (Fig. 3). Various cell types, including reported glial and neuronal subpopulations (Romero et al., 2000), were found to express GFP throughout the dorsal spinal cord (Fig. $3 A, C$ ). On the contrary, immunoreactivity to detect FLAG expression was similarly distributed but appeared as diffuse extracellular staining (Fig. 3B). In animals with T4 spinal transection and control GFP Adts injections, $\mathrm{CGRP}^{+}$fiber immunostaining in dorsal horns labeled characteristic dense bands in laminas I and II with some sprouts extending to lamina III and medial laminas IV and V (Fig. 3D). The spatial extent of exogenous NGF-FLAG immunostaining throughout the dorsal horns and columns mirrored the increased density of $\mathrm{CGRP}^{+}$fiber immunostaining (Fig. 3E). The widespread and somewhat punctate immunostaining of CGRP ${ }^{+} \mathrm{fi}^{-}$ bers with local NGF overexpression represents massive amounts of sprouting axons cut in cross section. The CGRP ${ }^{+}$fibers in Sema3a Adts-injected cords generally appeared similar to GFP Adts controls, with an apparent reduction in density (Fig. $3 F$ ).

To establish whether the immunohistochemical localization of transgene proteins actually reflected increased expression, Western blot analysis (Fig. 4) confirmed conspicuous elevation of exogenous GFP ( $\sim 35 \mathrm{kDa}), \mathrm{NGF}(\sim 14 \mathrm{kDa})$, and/or Sema3a $(\sim 97.4 \mathrm{kDa})$ within experimental groups, as predicted based on previous results (Romero et al., 2000; Tang et al., 2004). The presence of GFP was also observed in the same spinal cords treated with Sema3a, because this virus expressed both independently.

\section{Effects of intraspinal NGF versus GFP versus}

Sema3a overexpression

Because NGF overexpression at T5/6 after T4 transection did not elevate hypertensive dysreflexia during noxious CRD compared with GFP Adts-injected controls at any spinal level (Fig. 2), the T5/6 level was not quantified histologically. Even so, compared 


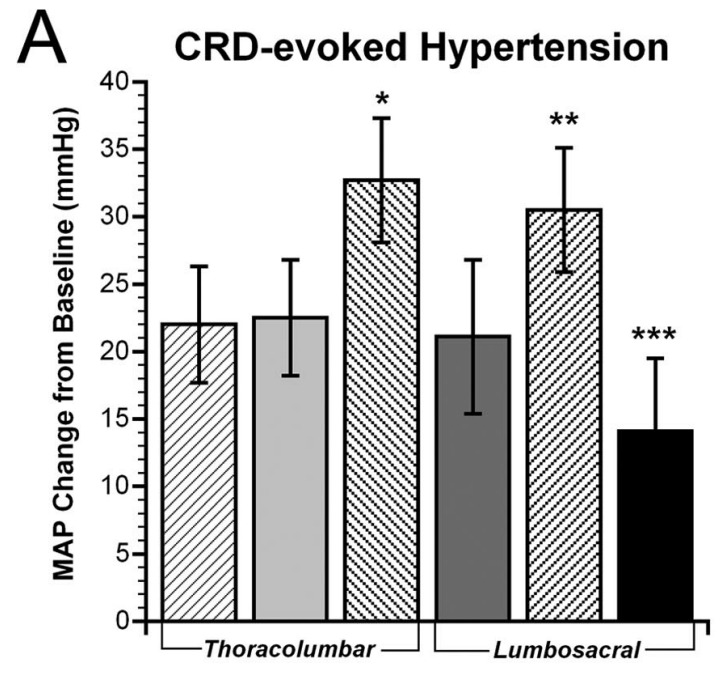

Treatment/Spinal Level

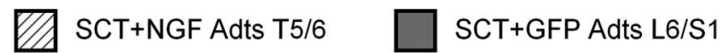

SCT+GFP Adts T13/L1 $Z$ SCT+NGF Adts L6/S1

SCT+NGF Adts T13/L1 $\square$ SCT+Sema3a Adts L6/S1

B

\section{CRD-evoked Bradycardia}

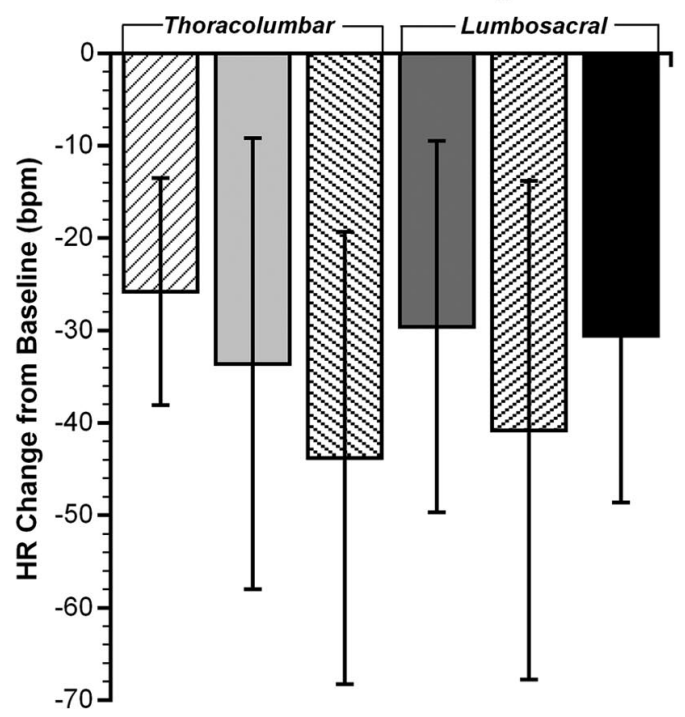

Figure 2. Quantitative assessments of autonomic dysreflexia severity in injured rats among treatment groups. $\boldsymbol{A}$, Two weeks after T4 spinal cord transection (SCT), NGF overexpression in either $\mathrm{T} 13 / \mathrm{L} 1$ or L6/S1 significantly increased hypertension versus GFP overexpression at the same levels, as well as NGF Adts injections at T5/6. Conversely, Sema3a overexpression in L6/S1 significantly reduced noxious CRD-induced hypertension. $\boldsymbol{B}$, The bradycardia manifested in response to visceral pain in all rats with SCT was significantly $(p<0.0001)$ different from and diametrically opposite to tachycardia observed in uninjured rats (data not shown). The magnitude of bradycardia across the groups mirrored the extent of hypertension, despite the high variability in $\mathrm{HR}$ within treatment groups. Bars represent means $\pm S D$. ${ }^{*} p<0.01$ versus GFP Adts T13/L1 and NGF Adts T5/6; ${ }^{* *} p<0.005$ versus GFP Adts L6/S1; ${ }^{* * *} p<0.01$ versus GFP Adts L6/S1 and $p<0.0001$ versus NGF Adts L6/S1.

with GFP controls (Fig. 5A), copious $\mathrm{CGRP}^{+}$fiber sprouting was seen throughout the dorsal spinal cord (Fig. $5 B$ ), and numerous aberrant $\mathrm{CGRP}^{+}$fiber sprouts extended proximal to prelabeled sympathetic preganglionic neurons in the IML (Fig. 5C). Again, this reinforces the physiological data that neither NGF nor $\mathrm{CGRP}^{+}$fibers directly alter the sympathetic discharge in response to noxious CRD but does not rule out the likelihood that noxious stimuli applied to the T5/6 dermatome would augment autonomic dysreflexia to some extent.

Compared with T5/6 NGF Adts injections, similar CGRP ${ }^{+}$ fiber distribution was seen throughout T13/L1 $(n=9)$ and L6/S1 $(n=8)$ dorsal columns and gray matter with NGF overexpression, including lamina $\mathrm{X}$ surrounding the central canal (Fig. $6 A, B)$. An ANOVA across the critical spinal levels (T13/L1, L6/ S1) and treatment groups (GFP, NGF, Sema3a Adts) revealed a significant difference in the extent of $\mathrm{CGRP}^{+}$fiber density in the dorsal horns $\left(F_{(4,38)}=21.107 ; p<0.001\right)$. In rats injected with GFP Adts into T13/L1 $(n=5)$ or L6/S1 $(n=9)$ spinal cord segments, the $\mathrm{CGRP}^{+}$areal coverage in the dorsal gray matter chosen for quantification (Fig. $6 A, B$ ) was $\sim 10-13 \%$, respectively (Fig. 7). Compared with GFP controls, NGF overexpression significantly increased $\mathrm{CGRP}^{+}$fiber immunoreactivity up to $20 \%$ at T13/L1 and L6/S1 segments (Fig. 7).

The distribution of CGRP ${ }^{+}$fibers in Sema3a Adts-injected L6/S1 cords $(n=14)$ generally appeared similar to GFP Adts controls (Fig. $6 \mathrm{~B}$ ). However, immunostaining in the dorsal horns was less evident, particularly in the deeper laminas (Fig. 6C). Quantitatively, the spatial extent of CGRP ${ }^{+}$staining in the lumbosacral dorsal horns was significantly reduced compared with GFP controls (Fig. 7), demonstrating that Sema3a overexpression decreased the amount of local spontaneous $\mathrm{CGRP}^{+}$fiber sprouting into the spinal cord after T4 spinal transection.

The combined physiological and histological data indicate that after complete spinal cord injury, the site-directed overexpression of NGF in lumbosacral spinal levels significantly increased both CRD-evoked dysreflexic hypertension and CGRP ${ }^{+}$ fiber sprouting, whereas overexpression of Sema3a significantly reduced sprouting and hypertension. Quantitatively, there was a significant positive correlation between the extent of CGRP ${ }^{+}$ fiber distribution in the thoracolumbar or lumbosacral spinal levels and the severity of autonomic dysreflexia among GFP, NGF, and Sema3a Adts-injected groups (Fig. 8).

Similar quantification of substance $\mathrm{P}\left(\mathrm{SP}^{+}\right)$fiber densities in the dorsal horns of adjacent sections (Fig. 9) revealed a significant difference across treatment groups between spinal levels $\left(F_{(4,38)}=8.901 ; p<0.01\right)$. Whereas NGF overexpression at $\mathrm{T} 13 / \mathrm{L} 1$ and L6/S1 significantly increased $\mathrm{SP}^{+}$fiber density by $\sim 50 \%$ compared with GFP Adts controls (Fig. 9), Sema3a Adts injections did not lower $\mathrm{SP}^{+}$immunoreactivity in lumbosacral levels below that of the normal density. This is similar to our previous observations demonstrating that Sema3a does not induce retraction of established (normal) fiber densities but only prevents the sprouting of these axons (Tang et al., 2004). Moreover, extremely low baseline values of $\mathrm{SP}^{+}$fiber immunoreactive densities in GFP Adts-injected spinal cords $(\sim 4 \%)$, more than twofold less compared with CGRP ${ }^{+}$(compare with Fig. 7), make such comparisons difficult. In addition, the high degree of variability in thresholding of extremely punctate $\mathrm{SP}^{+}$fiber immunostaining in cross sections also prevents precise interpretation of Sema3a overexpression on $\mathrm{SP}^{+}$fiber density.

\section{Discussion}

Although the etiology of autonomic dysreflexia is not entirely understood, injury-induced elevations in spinal levels of nerve growth factor (Bennett et al., 1999; Brown et al., 2004) and the subsequent sprouting of $\mathrm{CGRP}^{+}$afferent fibers in the thoracolumbar spinal cord (McNeill et al., 1990, 1991) are reported to be 
contributing factors underlying the development of experimental autonomic dysreflexia (Krenz and Weaver, 1998; Krenz et al., 1999; Weaver et al., 2001; Marsh et al., 2002). Therefore, we precisely manipulated injury-induced $\mathrm{CGRP}^{+}$fiber sprouting in the dorsal horns with bilateral microinjections of well characterized recombinant adenovirus encoding growth-promoting NGF (Romero et al., 2000, 2001; Tang et al., 2004). The principal goal was to target NGF overexpression in the dorsal gray matter of selected spinal segments, caudal to a complete T4 transection, and genetically modulate sites mediating dysreflexic responses to noxious CRD 2 weeks after injury (Maiorov et al., 1998).

It was our hypothesis that, in a model of noxious CRD-induced dysreflexia, increased NGF-mediated sprouting of sacral nociceptive C-fiber afferents after complete thoracic spinal cord injury (de Groat et al.,

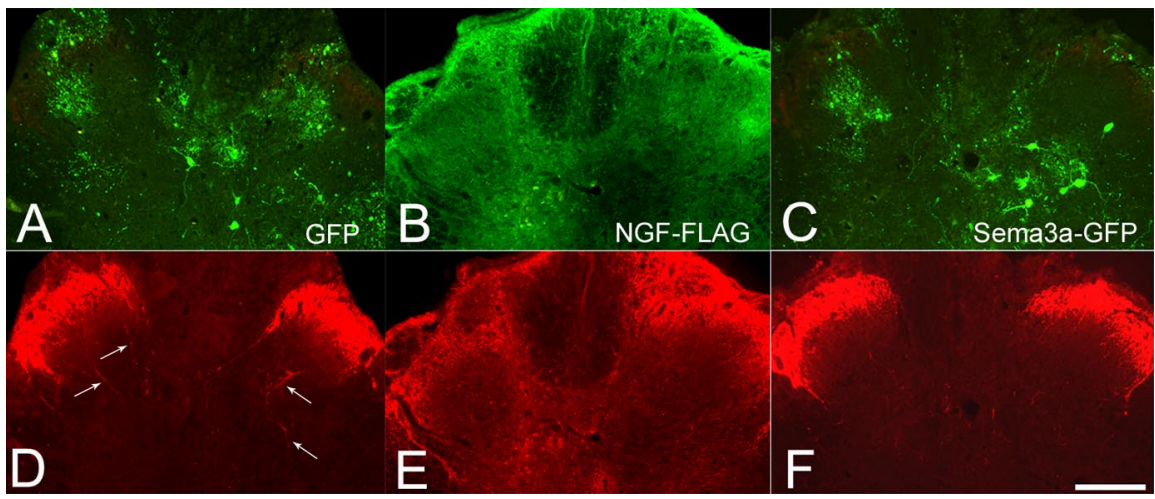

Figure 3. $A-C$, Series of photomicrographs demonstrating how sections were designated for $\mathrm{CGRP}^{+}$fiber immunostaining and quantification at sites of bilateral adenovirus microinjections that encoded control GFP (A), NGF tagged with the FLAG epitope $(\boldsymbol{B})$, or Sema3a that coexpressed GFP (C). The GFP localization was intracellular, particularly in glial and neuronal subpopulations, whereas exogenous FLAG immunoreactivity appeared as widespread extracellular staining. Dual immunofluorescent images in the bottom row show the corresponding spatial extent of $\mathrm{CGRP}^{+}{ }^{+}$immunostaining in $\mathrm{L} 6 / \mathrm{S} 1$ segments with overexpression of GFP (D), $\operatorname{NGF}(\boldsymbol{E})$, or Sema3a $(\boldsymbol{F})$. Note the modest CGRP ${ }^{+}$fiber sprouting seen in GFP controls (arrows in $\boldsymbol{D}$ ). Conversely, the widespread and punctate immunostaining of $\mathrm{CGRP}^{+}$fibers mirrored diffuse immunolocalization of exogenous NGF-FLAG throughout the dorsal horns and even dorsal columns. Scale bar: (in $\boldsymbol{F}) \boldsymbol{A}-\boldsymbol{F}, 200 \mu \mathrm{m}$. 1990) drives larger numbers of propriospinal projection neurons located in the dorsal commissural nucleus (Matsushita, 1998; Wang et al., 1998; Petkó and Antal, 2000; Vizzard, 2000). This, in turn, relays the signal to rostral sympathetically correlated interneurons that activate sympathetic preganglionic neurons in the IML to elicit hypertension (Chau et al., 2000; Tang et al., 2003). It is reported that after spinal cord injury, increased CGRP-immunoreactive fibers colocalize with GAP-43 indicating sprouting of nociceptive primary afferent fibers and not neuropeptide upregulation (Ondarza et al., 2003). Accordingly, once we confirmed the critical spinal levels physiologically with subsequent immunohistochemical analyses for $\mathrm{CGRP}^{+}$afferent fiber sprouting (Krenz et al., 1999; Weaver et al., 2001), we used recombinant adenovirus to overexpress Sema3a after injury to prevent $\mathrm{CGRP}^{+}$fiber sprouting (Tang et al., 2004) and mitigate dysreflexic hypertension.

In accordance with our findings, we showed previously that overexpression of NGF within the spinal cord directly increased sprouting of $\mathrm{CGRP}^{+}$and $\mathrm{SP}^{+}$axons without altering neuropeptide expression or sprouting of other sensory axon populations (Tang et al., 2004). Moreover, we reported that overexpression of Sema3a, a repulsive guidance molecule for these fibers, reduces sprouting of CGRP ${ }^{+}$and $\mathrm{SP}^{+}$axons compared with overexpression of control GFP (Tang et al., 2004). Sema3a is a potent inhibitor of NGF-responsive nociceptive C-fiber growth during development (He and Tessier-Lavigne, 1997; Yu and Kolodkin, 1999), as well as sprouting into the mature spinal cord (Tang et al., 2004). Our anatomical studies showed that the area coverage of $\mathrm{CGRP}^{+}$fibers in the lumbosacral dorsal horns was significantly decreased with Sema3a overexpression compared with GFP controls. Regression analysis confirmed that the magnitude of CRDinduced hypertensive changes was significantly correlated with the density of CGRP ${ }^{+}$fibers.

Subpopulations of $\mathrm{CGRP}^{+}$fibers sprouting distal to spinal cord injury sites are reported to be nociceptive primary afferents that could be anatomical substrates for chronic pain syndromes (Christensen and Hulsebosch, 1997a; Ondarza et al., 2003). Immuno-localization of CGRP is reported primarily in $\mathrm{A} \delta$ - and C-fiber afferent projections (Lawson et al., 1993, 1996), and $\mathrm{CGRP}^{+}$fibers can be labeled with various neurotransmitter markers. Approximately 50\% of the small-diameter neurons in
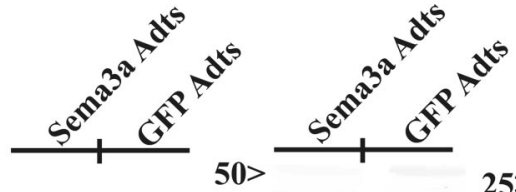

25>
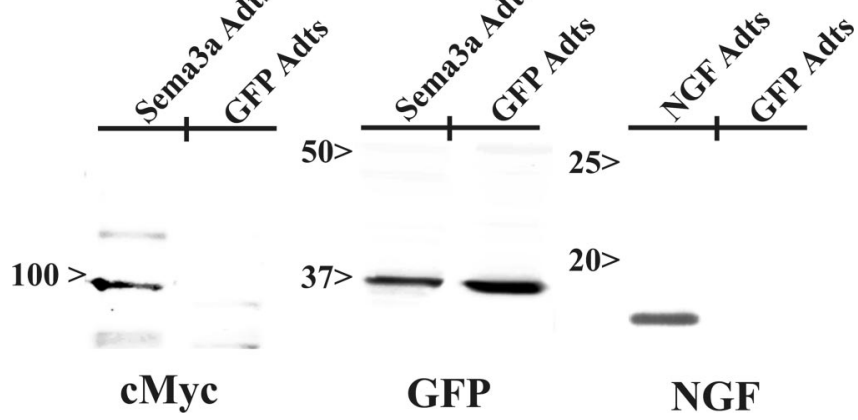

20>

Figure 4. Western blot analyses confirm increased expression of transgene protein after injections of Sema3a Adts, GFP Adts, or NGF Adts into spinal cords. Sema3a was identified at $\sim 97 \mathrm{kDa}$ using an anti-myc antibody to recognize this C-terminal tag on the protein. GFP expression was observed in both Sema3a Adts- and control-injected cords. Likewise, NGF (17 kDa) was identified using a antibody specific for NGF.

dorsal root ganglia are nonpeptidergic, they do not respond to NGF, and their contribution to eliciting dysreflexic hypertension remains uncertain. The remaining small-diameter population are tyrosine receptor kinase A (trkA)-expressing CGRP ${ }^{+}$nociceptive neurons (Averill et al., 1995; Molliver and Snider, 1997), whereas a subpopulation of CGRP ${ }^{+}$fibers coexpress SP (Snider and McMahon, 1998).

Importantly, a lack of significant $\mathrm{SP}^{+}$fiber sprouting in parallel with increased $\mathrm{CGRP}^{+}$fiber distribution has been reported after injury in a similar rat model of autonomic dysreflexia (Marsh and Weaver, 2004), comparable with our current findings. Although in our original dorsal rhizotomy model, the sprouting of peptidergic nociceptive axons correlated with hyperalgesia and allodynia, it remains unknown whether such abnormal pain responses contribute to autonomic dysreflexia. What is apparent is that these NGF-responsive nociceptive axons contribute to the onset of autonomic dysreflexia, independent of other nonpeptidergic nociceptive axons.

Two main bodies of evidence exist that increased NGF production is related to intraspinal plasticity that contributes to the development of autonomic dysreflexia. First, 2 weeks after T4 spinal transection, there is increased sprouting of NGF- 


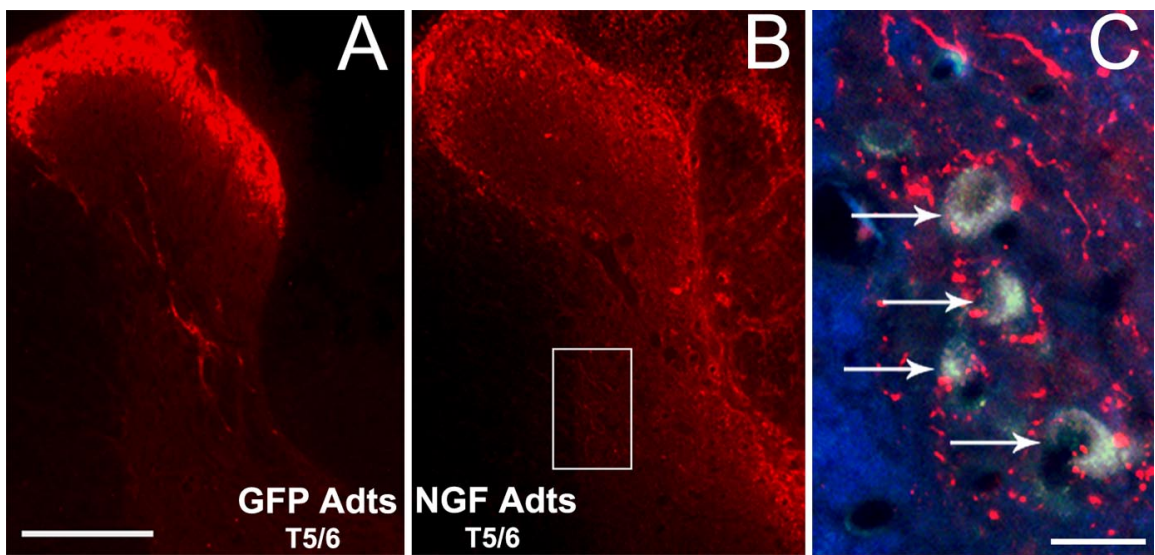

Figure 5. Photomicrographs showing $\mathrm{CGRP}^{+}$fiber immunostaining at mid-thoracic spinal levels that received bilateral Adts injections. $A, B$, Compared with control GFP Adts injections $(\boldsymbol{A})$, the overexpression of NGF at T5/6 $(\boldsymbol{B})$ elicited profuse $\mathrm{CGRP}^{+}$fiber sprouting into the dorsal spinal cord after $15 \mathrm{~d}$ postinjury. $C$, Superimposed dual-immunofluorescence, high-magnification images of NGF Adts-injected lateral gray matter (boxed region in $\boldsymbol{B}$ ) shows numerous (GRP ${ }^{+}$fibers extending proximal to sympathetic preganglionic neurons (arrows) prelabeled with FluoroGold. This was not observed in T5/6 cords injected with GFP Adts (data not shown). Scale bars: (in $\boldsymbol{A}, \boldsymbol{C}) \boldsymbol{A}, \boldsymbol{B}, 200 \mu \mathrm{m} ; \boldsymbol{C}, 20 \mu \mathrm{m}$.
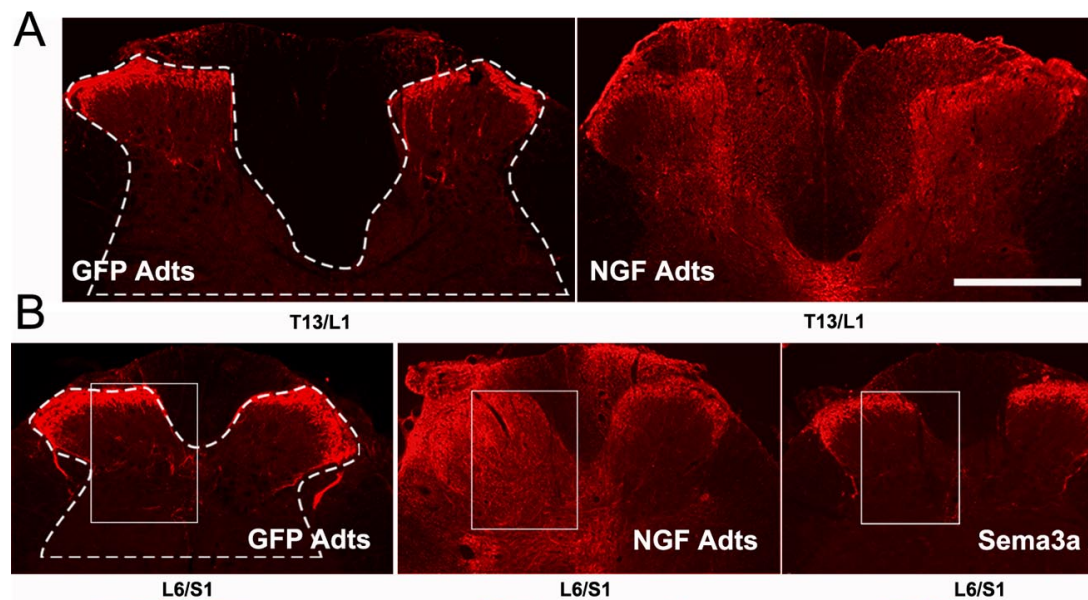

T13/L1
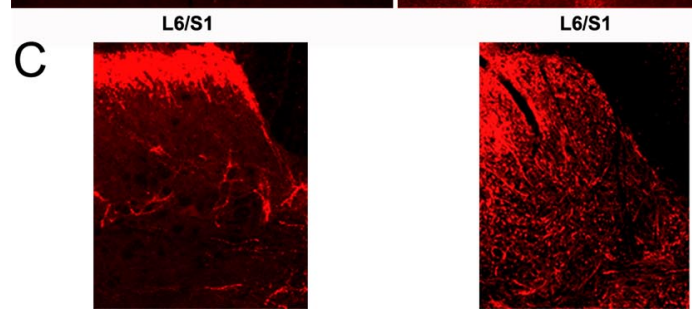

NGF Adts

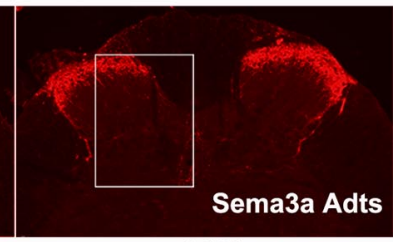

Figure 6. $\quad \boldsymbol{A}, \boldsymbol{B}$, Photomicrographs showing $\mathrm{GGP}^{+}$fiber immunostaining at thoracolumbar $(\boldsymbol{A})$ or lumbosacral $(\boldsymbol{B})$ spinal levels that received bilateral Adt injections. $C$, Higher magnifications demonstrate $\mathrm{CGRP}^{+}{ }^{+}$immunostaining in $\mathrm{L} 6 / \mathrm{S} 1$ dorsal horns (boxed regions in $\boldsymbol{B}$ ). Compared with control GFP Adts, NGF overexpression in the thoracolumbar and lumbosacral spinal cord induced $\mathrm{CGRP}^{+}$fiber sprouting throughout the dorsal horns and even dorsal columns. Conversely, Sema3a Adts reduced post-traumatic aberrant CGRP ${ }^{+}$fiber sprouting into the $L 6 / S 1$ dorsal horns. Scale bar: (in $\left.A\right) A, B, 500 \mu \mathrm{m} ; C, 200 \mu \mathrm{m}$.

responsive $\mathrm{CGRP}^{+}$primary afferents in laminas III-V of the thoracic and lumbar dorsal horns (Krenz and Weaver, 1998). Second, NGF-responsive C-fibers innervating the pelvic viscera undergo significant changes after spinal cord injury, including expansion of the territory occupied by their central terminals (de Groat et al., 1990) and an increase in the cross-sectional area of the somata in the L6/S1 dorsal root ganglia (Kruse et al., 1995).

NGF neutralization after experimental spinal cord injury elicits changes in $\mathrm{CGRP}^{+}$fiber density in the dorsal gray matter (Christensen and Hulsebosch, 1997b). Notably, the intrathecal administration of NGF antibodies (Krenz et al., 1999) or trkA
Sema3a Adts

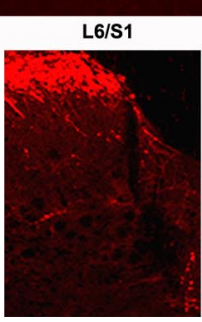

fusion proteins (Marsh et al., 2002) reduce both injury-induced $\mathrm{CGRP}^{+}$fiber density in laminas III-V, as well as noxious CRD-induced dysreflexia. With reference to our findings, such effects may relate to caudal transport of NGF antibodies or trkA fusion proteins in CSF to lower lumbosacral regions that were not examined. For instance, intrathecal delivery of NGF antibodies after spinal cord transection reduces elevated NGF protein levels in L6 and S1 dorsal root ganglia and spinal cord (Seki et al., 2002). Such treatment also reduces bladder hyper-reflexia in spinal injured rats, known to be mediated by $\mathrm{CGRP}^{+}$, trkA $^{+} \mathrm{C}$-fiber afferents (Cheng et al., 1995; Bennett et al., 1996).

Based on electrophysiological measurements, CRD activates sympathetically correlated neurons in the T10 segment in acute and chronic spinal-transected rats (Chau et al., 2000; Krassioukov et al., 2002). In chronically spinal-transected rats, noxious CRD elicits greater increases in arterial pressure, possibly mediated by hyperexcitability of expanded sympathetically correlated interneurons. This suggests that spinal circuits undergo significant plastic changes manifested in chronic stages of spinal cord injury. Neurons of the dorsal commissural nucleus in the T13/L1 and L6/S1 regions respond to input from pelvic viscera (Ness and Gebhart, 1987; Traub et al., 1993) and project axons rostrally in gray matter (Wang et al., 1999) as well as the dorsal columns (Willis et al., 1999). Both types of relay extend the entire length of the cord and, therefore, may sprout collaterals to influence the activity of preganglionic sympathetic neurons in the IML (Llewellyn-Smith and Weaver, 2001) and dorsal commissural nucleus (Hosoya et al., 1994). In support of such an association, we found that microinjection of the anterograde tract tracer biotinylated dextran amine in the S1 dorsal horn of T4-transected animals versus uninjured controls labels significantly more propriospinal projections in thoracolumbar gray matter 2 weeks after injury (Rabchevsky, 2006).

Notably, experimental autonomic dysreflexia was not completely eliminated by impeding CGRP ${ }^{+}$fiber sprouting with Sema3a overexpression, similar to previous findings in which endogenous NGF was neutralized after complete T4 spinal transection (Krenz et al., 1999; Marsh et al., 2002). Although Sema3a overexpression significantly reduced overall $\mathrm{CGRP}^{+}$immunostaining below baseline, low levels of CRDinduced hypertension persisted. This implies that, in addition to sprouting of $\mathrm{CGRP}^{+}$fibers, spinal cord injury most likely provokes NGF-independent reorganization of propriospinal pathways as well. On the other hand, the high-affinity NGF trkA receptor is located on cholinergic propriospinal neurons in the 


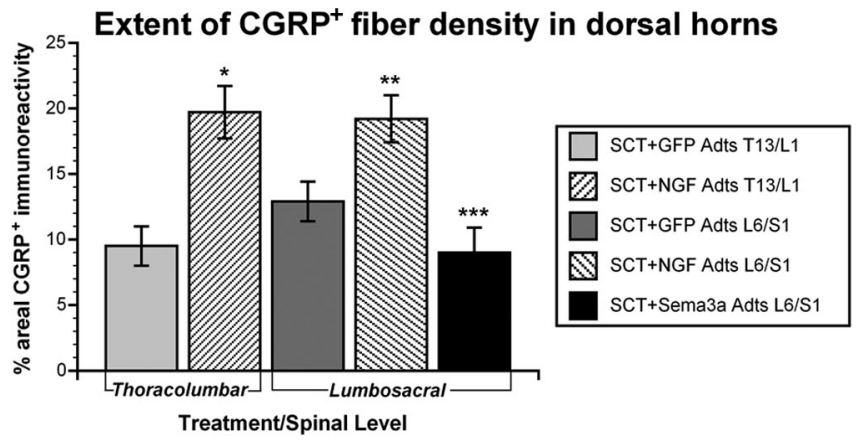

Figure 7. Quantitative spatial analysis of $\mathrm{CGRP}^{+}$fiber staining in dorsal horns (see dashed lines in Fig. $6 A, B$ ) of sections through Adts injection sites $15 \mathrm{~d}$ after spinal cord transection (SCT). NGF overexpression in thoracolumbar and lumbosacral regions significantly increased $\mathrm{CGRP}^{+}$fiber density compared with control GFP. In contrast, Sema3a Adts overexpression in lumbosacral levels significantly reduced (GRP ${ }^{+}$fiber density versus injured controls. Bars represent means \pm SD. ${ }^{*} p<0.001$ versus GFP Adts T13/L1; ${ }^{* *} p<0.005$ versus GFP Adts L6/S1; ${ }^{* * *} p<0.001$ versus GFP Adts $L 6 / S 1$ and $p<0.0001$ versus NGF Adts $L 6 / S 1$.

\section{Hypertension versus Sprouting}

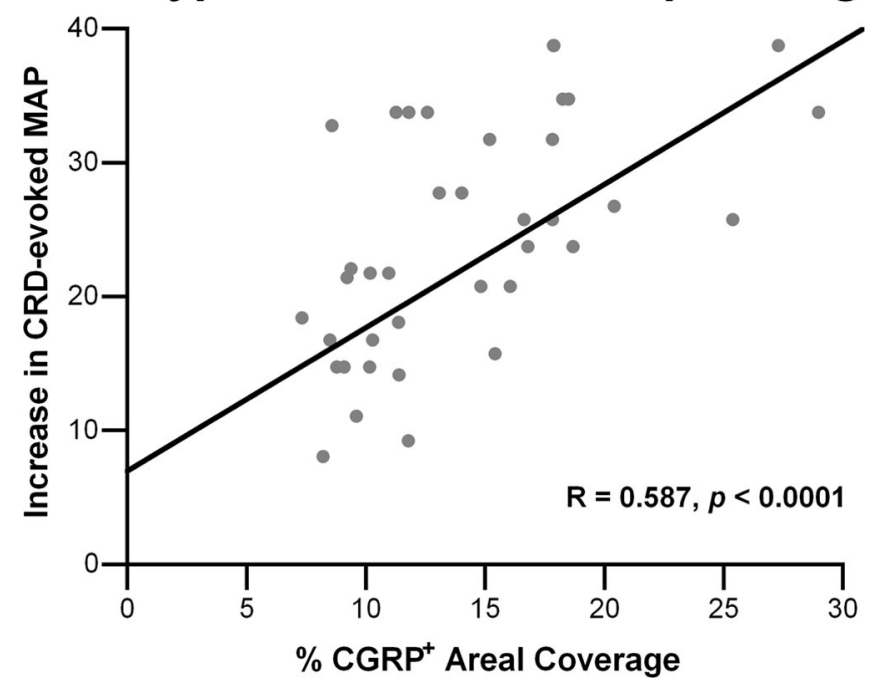

Figure 8. Linear regression analysis demonstrates a significant $(p<0.0001)$ positive correlation between the extent of noxious CRD-evoked hypertension and the density of CGRP ${ }^{+}$ fibers in dorsal horns among spinal rats overexpressing GFP, NGF, or Sema3a at the different spinal levels.

deep dorsal horn of the rat spinal cord (Michael et al., 1997), so the expansion of sacral-thoracic propriospinal projection may also be an NGF-dependent response to spinal injury. Such changes may provide a neural substrate for the amplification of discrete afferent signals (e.g., pressure sores, distended bladder or rectum), which elicit the synchronous discharge of sympathetic preganglionic neurons of the thoracic sympathetic chain, resulting in global sympathetic arousal including a potentially fatal hypertensive crisis. Additional studies will examine whether Sema3a can cause retraction of established connections and reverse dysreflexia that has already developed for potentially translational approaches for chronic injuries.

In conclusion, by regionally altering endogenous neural circuitry, we demonstrate a mechanistic link that ties noxious CRDinduced dysreflexic hypertension directly to changes in CGRP ${ }^{+}$ fiber sprouting. Intraspinal overexpression of chemorepulsive Sema3a significantly impeded such sprouting and mitigated hypertensive autonomic dysreflexia. The opposite conditions occur
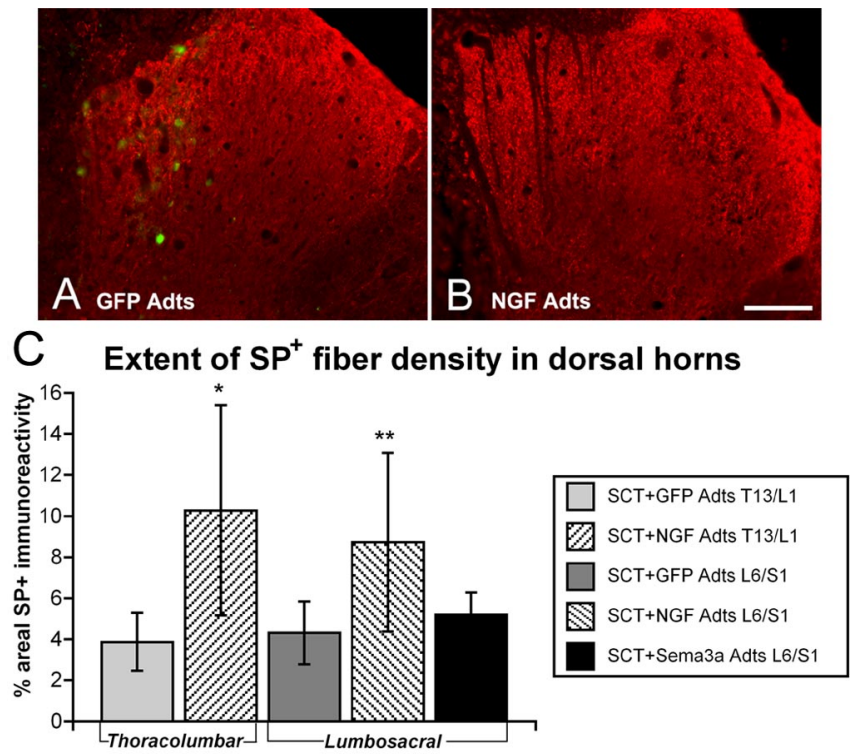

Treatment/Spinal Level

Figure 9. $A, B$, Photomicrographs showing $\mathrm{SP}^{+}$fiber staining in thoracolumbar dorsal horns injected with adenovirus encoding control GFP Adts $(\boldsymbol{A})$ versus NGF Adts $(\boldsymbol{B})$. Note transfected cells expressing GFP seen with dual immunofluorescence in $\boldsymbol{A}$. Similar to $\mathrm{CGRP}^{+}$fiber staining, NGF overexpression elicited profuse $\mathrm{SP}^{+}$fiber sprouting throughout the dorsal horns, although the staining is far less intense and more punctuate in this CGRP ${ }^{+}$fiber subpopulation. Scale bar: (in B) $200 \mu \mathrm{m}$. C, Quantitative spatial analysis of SP ${ }^{+}$immunostaining in dorsal horns of sections adjacent to those stained for (GRP $15 \mathrm{~d}$ after spinal cord transection (SCT). NGF overexpression significantly increased SP ${ }^{+}$fiber density compared with control GFP. In contrast, with Sema3a overexpression, there was no observable alteration in $\mathrm{SP}^{+}$fiber densities versus injured controls. Bars represent means $\pm S D .{ }^{*} p<0.01$ versus GFP Adts $\mathrm{T} 13 / \mathrm{L} 1$; ${ }^{* *} p<$ 0.05 versus GFP Adts $L 6 / S 1$ and Sema3a Adts L6/S1.

with NGF overexpression, demonstrating that axon guidance and inhibitory molecules can alter endogenous circuitry to modulate plasticity-induced autonomic pathophysiology after spinal cord injury. To illustrate the importance of these investigations, a recent survey of individuals with spinal cord injury ranked restoration of desired functions in order of importance to their quality of life (Anderson, 2004). The results showed that for quadriplegics and paraplegics, the restoration of arm and sexual function had highest priorities, respectively, but alleviating autonomic dysfunction (bowel, bladder, autonomic dysreflexia) was of higher importance than walking for both groups. The current study demonstrates that the neural plasticity that may underlie the development of autonomic dysreflexia after complete spinal cord injury can be altered by gene therapy and, perhaps, pharmacological agents that mimic plasticity-inhibiting agents. Importantly, such approaches may also influence intraspinal plasticity in other forms of dysfunction and/or recovery after spinal cord injury, such as chronic pain and locomotion.

\section{References}

Anderson CR, Edwards SL (1994) Intraperitoneal injections of Fluorogold reliably labels all sympathetic preganglionic neurons in the rat. J Neurosci Methods 53:137-141.

Anderson KD (2004) Targeting recovery: priorities of the spinal cord injured population. J Neurotrauma 21:1371-1383.

Averill S, McMahon SB, Clary DO, Reichardt LF, Priestley JV (1995) Immunocytochemical localization of trkA receptors in chemically identified subgroups of adult rat sensory neurons. Eur J Neurosci 7:1484-1494.

Bennett AD, Taglialatela G, Perez-Polo R, Hulsebosch CE (1999) NGF levels decrease in the spinal cord and dorsal root ganglion after spinal hemisection. NeuroReport 10:889-893.

Bennett DL, Dmietrieva N, Priestley JV, Clary D, McMahon SB (1996) trkA, 
CGRP and IB4 expression in retrogradely labelled cutaneous and visceral primary sensory neurones in the rat. Neurosci Lett 206:33-36.

Brown A, Ricci MJ, Weaver LC (2004) NGF message and protein distribution in the injured rat spinal cord. Exp Neurol 188:115-127.

Cameron AA, Smith GM, Randall DC, Brown DR, Rabchevsky AG (2004) Genetic manipulation of afferent fiber sprouting following spinal cord injury modulates the severity of autonomic dysreflexia. J Neurotrauma 21:1271.

Chau D, Johns DG, Schramm LP (2000) Ongoing and stimulus-evoked activity of sympathetically correlated neurons in the intermediate zone and dorsal horn of acutely spinalized rats. J Neurophysiol 83:2699-2707.

Cheng CL, Ma CP, de Groat WC (1995) Effect of capsaicin on micturition and associated reflexes in chronic spinal rats. Brain Res 678:40-48.

Christensen MD, Hulsebosch CE (1997a) Chronic central pain after spinal cord injury. J Neurotrauma 14:517-537.

Christensen MD, Hulsebosch CE (1997b) Spinal cord injury and anti-NGF treatment results in changes in CGRP density and distribution in the dorsal horn in the rat. Exp Neurol 147:463-475.

de Groat WC, Kawatani M, Hisamitsu T, Cheng CL, Ma CP, Thor K, Steers W, Roppolo JR (1990) Mechanisms underlying the recovery of urinary bladder function following spinal cord injury. J Auton Nerv Syst [Suppl] 30:S71-S77.

Finestone HM, Teasell RW (1993) Autonomic dysreflexia after brainstem tumor resection. A case report. Am J Phys Med Rehabil 72:395-397.

Harati Y (1997) Autonomic disorders associated with spinal cord injury. In: Clinical autonomic disorders, Ed 2 (Low PA, ed), pp 455-461. Philadelphia: Lippincott-Raven.

He TC, Zhou S, da Costa LT, Yu J, Kinzler KW, Vogelstein B (1998) A simplified system for generating recombinant adenoviruses. Proc Natl Acad Sci USA 95:2509-2514.

He Z, Tessier-Lavigne M (1997) Neuropilin is a receptor for the axonal chemorepellent Semaphorin III. Cell 90:739-751.

Hosoya Y, Nadelhaft I, Wang D, Kohno K (1994) Thoracolumbar sympathetic preganglionic neurons in the dorsal commissural nucleus of the male rat: an immunohistochemical study using retrograde labeling of cholera toxin subunit B. Exp Brain Res 98:21-30.

Karlsson AK (1999) Autonomic dysreflexia. Spinal Cord 37:383-391.

Krassioukov AV, Weaver LC (1995) Episodic hypertension due to autonomic dysreflexia in acute and chronic spinal cord-injured rats. Am J Physiol 268:H2077-H2083.

Krassioukov AV, Johns DG, Schramm LP (2002) Sensitivity of sympathetically correlated spinal interneurons, renal sympathetic nerve activity, and arterial pressure to somatic and visceral stimuli after chronic spinal injury. J Neurotrauma 19:1521-1529.

Krenz NR, Weaver LC (1998) Sprouting of primary afferent fibers after spinal cord transection in the rat. Neuroscience 85:443-458.

Krenz NR, Meakin SO, Krassioukov AV, Weaver LC (1999) Neutralizing intraspinal nerve growth factor blocks autonomic dysreflexia caused by spinal cord injury. J Neurosci 19:7405-7414.

Kruse MN, Bray LA, de Groat WC (1995) Influence of spinal cord injury on the morphology of bladder afferent and efferent neurons. J Auton Nerv Syst 54:215-224.

Lawson SN, Perry MJ, Prabhakar E, McCarthy PW (1993) Primary sensory neurones: neurofilament, neuropeptides, and conduction velocity. Brain Res Bull 30:239-243.

Lawson SN, McCarthy PW, Prabhakar E (1996) Electrophysiological properties of neurones with CGRP-like immunoreactivity in rat dorsal root ganglia. J Comp Neurol 365:355-366.

Lindan R, Joiner E, Freehafer AA, Hazel C (1980) Incidence and clinical features of autonomic dysreflexia in patients with spinal cord injury. Paraplegia 18:285-292.

Llewellyn-Smith IJ, Weaver LC (2001) Changes in synaptic inputs to sympathetic preganglionic neurons after spinal cord injury. J Comp Neurol 435:226-240.

Maiorov DN, Krenz NR, Krassioukov AV, Weaver LC (1997) Role of spinal NMDA and AMPA receptors in episodic hypertension in conscious spinal rats. Am J Physiol 273:H1266-H1274.

Maiorov DN, Fehlings MG, Krassioukov AV (1998) Relationship between severity of spinal cord injury and abnormalities in neurogenic cardiovascular control in conscious rats. J Neurotrauma 15:365-374.

Marsh DR, Weaver LC (2004) Autonomic dysreflexia, induced by noxious or innocuous stimulation, does not depend on changes in dorsal horn substance p. J Neurotrauma 21:817-828.

Marsh DR, Wong ST, Meakin SO, MacDonald JI, Hamilton EF, Weaver LC (2002) Neutralizing intraspinal nerve growth factor with a trkA-IgG fusion protein blocks the development of autonomic dysreflexia in a clipcompression model of spinal cord injury. J Neurotrauma 19:1531-1541.

Matsushita M (1998) Ascending propriospinal afferents to area X (substantia grisea centralis) of the spinal cord in the rat. Exp Brain Res 119:356-366.

McNeill DL, Carlton SM, Coggeshall RE, Hulsebosch CE (1990) Denervation-induced intraspinal synaptogenesis of calcitonin generelated peptide containing primary afferent terminals. J Comp Neurol 296:263-268.

McNeill DL, Carlton SM, Hulsebosch CE (1991) Intraspinal sprouting of calcitonin gene-related peptide containing primary afferents after deafferentation in the rat. Exp Neurol 114:321-329.

Michael GJ, Kaya E, Averill S, Rattray M, Clary DO, Priestley JV (1997) TrkA immunoreactive neurones in the rat spinal cord. J Comp Neurol 385:441-455.

Molliver DC, Snider WD (1997) Nerve growth factor receptor TrkA is down-regulated during postnatal development by a subset of dorsal root ganglion neurons. J Comp Neurol 381:428-438.

Ness TJ, Gebhart GF (1987) Characterization of neuronal responses to noxious visceral and somatic stimuli in the medial lumbosacral spinal cord of the rat. J Neurophysiol 57:1867-1892.

Ondarza AB, Ye Z, Hulsebosch CE (2003) Direct evidence of primary afferent sprouting in distant segments following spinal cord injury in the rat: colocalization of GAP-43 and CGRP. Exp Neurol 184:373-380.

Pascual JI, Insausti R, Gonzalo LM (1993) Urinary bladder innervation in male rat: termination of primary afferents in the spinal cord as determined by transganglionic transport of WGA-HRP. J Urol 150:500-504.

Petkó M, Antal M (2000) Propriospinal afferent and efferent connections of the lateral and medial areas of the dorsal horn (laminae I-IV) in the rat lumbar spinal cord. J Comp Neurol 422:312-325.

Rabchevsky AG (2006) Segmental organization of spinal reflexes mediating autonomic dysreflexia after spinal cord injury. Prog Brain Res 152:265-274.

Rabchevsky AG, Fugaccia I, Sullivan PG, Scheff SW (2001) Cyclosporin A treatment following spinal cord injury to the rat: behavioral effects and stereological assessment of tissue sparing. J Neurotrauma 18:513-522.

Rabchevsky AG, Fugaccia I, Sullivan PG, Blades DA, Scheff SW (2002) Efficacy of methylprednisolone therapy for the injured rat spinal cord. J Neurosci Res 68:7-18.

Romero MI, Smith GM (1998) Adenoviral gene transfer into the normal and injured spinal cord: enhanced transgene stability by combined administration of temperature-sensitive virus and transient immune blockade. Gene Ther 5:1612-1621.

Romero MI, Rangappa N, Li L, Lightfoot E, Garry MG, Smith GM (2000) Extensive sprouting of sensory afferents and hyperalgesia induced by conditional expression of nerve growth factor in the adult spinal cord. J Neurosci 20:4435-4445.

Romero MI, Rangappa N, Garry MG, Smith GM (2001) Functional regeneration of chronically injured sensory afferents into adult spinal cord after neurotrophin gene therapy. J Neurosci 21:8408-8416.

Seki S, Sasaki K, Fraser MO, Igawa Y, Nishizawa O, Chancellor MB, de Groat WC, Yoshimura N (2002) Immunoneutralization of nerve growth factor in lumbosacral spinal cord reduces bladder hyperreflexia in spinal cord injured rats. J Urol 168:2269-2274.

Snider WD, McMahon SB (1998) Tackling pain at the source: new ideas about nociceptors. Neuron 20:629-632.

Snow JC, Sideropoulos HP, Kripke BJ, Freed MM, Shah NK, Schlesinger RM (1978) Autonomic hyperreflexia during cystoscopy in patients with high spinal cord injuries. Paraplegia 15:327-332.

Tang X, Neckel ND, Schramm LP (2003) Locations and morphologies of sympathetically correlated neurons in the $\mathrm{T}(10)$ spinal segment of the rat. Brain Res 976:185-193. 
Tang XQ, Tanelian DL, Smith GM (2004) Semaphorin3A inhibits nerve growth factor-induced sprouting of nociceptive afferents in adult rat spinal cord. J Neurosci 24:819-827.

Traub RJ, Herdegen T, Gebhart GF (1993) Differential expression of c-fos and c-jun in two regions of the rat spinal cord following noxious colorectal distention. Neurosci Lett 160:121-125.

Vizzard MA (2000) Increased expression of spinal cord Fos protein induced by bladder stimulation after spinal cord injury. Am J Physiol Regul Integr Comp Physiol 279:R295-R305.

Wang CC, Willis WD, Westlund KN (1999) Ascending projections from the area around the spinal cord central canal: a Phaseolus vulgaris leucoagglutinin study in rats. J Comp Neurol 415:341-367.

Wang HF, Shortland P, Park MJ, Grant G (1998) Retrograde and transganglionic transport of horseradish peroxidase-conjugated cholera toxin $\mathrm{B}$ subunit, wheatgerm agglutinin and isolectin B4 from Griffonia simplicifolia $\mathrm{I}$ in primary afferent neurons innervating the rat urinary bladder. Neuroscience 87:275-288.

Weaver LC, Verghese P, Bruce JC, Fehlings MG, Krenz NR, Marsh DR (2001) Autonomic dysreflexia and primary afferent sprouting after clipcompression injury of the rat spinal cord. J Neurotrauma 18:1107-1119. Willis WD, Al-Chaer ED, Quast MJ, Westlund KN (1999) A visceral pain pathway in the dorsal column of the spinal cord. Proc Natl Acad Sci USA 96:7675-7679.

Yu HH, Kolodkin AL (1999) Semaphorin signaling: a little less per-plexin. Neuron 22:11-14.

Zagon A, Smith AD (1993) Monosynaptic projections from the rostral ventrolateral medulla oblongata to identified sympathetic preganglionic neurons. Neuroscience 54:729-743. 